\title{
Relaxation Dynamics at Different Time Scales in Electrostatic Complexes: Time-Salt Superposition
}

\author{
Evan Spruijt, ${ }^{1, *}$ Joris Sprakel, ${ }^{2}$ Marc Lemmers, ${ }^{1}$ Martien A. Cohen Stuart, ${ }^{1}$ and Jasper van der Gucht ${ }^{1}$ \\ ${ }^{1}$ Laboratory of Physical Chemistry and Colloid Science, Wageningen University, Dreijenplein 6, \\ 6703 HB Wageningen, The Netherlands \\ ${ }^{2}$ School of Engineering and Applied Sciences, Harvard University, 9 Oxford Street, Cambridge, O2138 Massachusetts, USA
}

(Received 15 June 2010; published 8 November 2010)

In this Letter we show that in the rheology of electrostatically assembled soft materials, salt concentration plays a similar role as temperature for polymer melts, and as strain rate for soft solids. We rescale linear and nonlinear rheological data of a set of model electrostatic complexes at different salt concentrations to access a range of time scales that is otherwise inaccessible. This provides new insights into the relaxation mechanisms of electrostatic complexes, which we rationalize in terms of a microscopic mechanism underlying salt-enhanced activated processes.

Electrostatically driven assemblies are ubiquitous in natural and man-made soft materials. In nature, attractive electrostatic forces play an important role in the condensation of DNA with histones [1], in the binding of peripheral proteins to membranes [2] and in the exceptional thermal stability of enzymes in thermophilic bacteria [3]. Nature's approaches for electrostatic assembly have been mimicked using synthetic building blocks to create photonic materials [4], self-healing gels [5], and multilayered capsules of various sizes and possible cargos [6]. Despite the plethora of phenomena where electrostatic attractions are relevant, it remains poorly understood how environmental factors, such as ionic strength, influence the dynamics of these complexes. Moreover, there are no clear methods available to experimentally analyze these effects.

In this Letter, we propose a new approach to study the dynamics of electrostatic complexes. We show that probing the rheological behavior of a model electrostatic complex at different ionic strengths is equivalent to probing the behavior at different time scales. This allows for superimposing rheological data at different salt concentrations, which together with the proposed model leads to a new way to systematically investigate the effects of temperature and salt concentration on relaxation behavior and mechanical properties of these complexes.

Superposition is used as a tool in rheology to probe dynamical processes in a wide variety of soft materials, thereby extending our understanding of their dynamics. For example, time-temperature superposition [7] relies on the acceleration of all activated processes at high temperatures, enabling probing of longer effective time scales at high temperatures. Analogously, strain-rate-frequency superposition allows one to probe low-frequency structural relaxation of soft solids, such as concentrated suspensions and foams, by enhancement of activated processes by external force [8]. In this Letter, we apply a similar approach to study the dynamics of a yet underrepresented class of soft materials: electrostatic complexes. In addition to the model materials presented here, our approach could easily be extended to other electrostatic complexes, both natural and man made, to help elucidate unresolved issues in the dynamics of electrostatic complexes.

We use complexes of two equally long and oppositely charged polyelectrolytes, namely, poly(acrylic acid), a polyanion (effective $p K_{a} 5.0-5.5$ for $c_{\text {salt }}=2.0-0.10 \mathrm{M}$ ), and $\operatorname{poly}(N, N$-dimethylaminoethyl methacrylate), a polycation (effective $p K_{a}$ 8.0-7.5). The phase behavior of these complexes in aqueous monovalent electrolyte solutions has been worked out experimentally [9]. We use polyelectrolytes with a degree of polymerization of $20,50,150$, or 510 $(\mathrm{PDI}<1.2)$ at $p \mathrm{H} 6.5$ where the polycation and polyanion have equal charge densities. When mixed together, the mixture phase separates into a dilute phase and a dense, viscoelastic phase that contains about $70 \mathrm{wt} \%$ water. Our polyelectrolyte complexes are prepared at different salt concentrations but always at 1:1 stoichiometry of polymeric charges. In addition, another set of polyelectrolytes, synthesized by atom transfer radical polymerization, with a degree of polymerization of 100 (PDI $<1.1$ ) is used [10]. These polyelectrolytes contain no $p \mathrm{H}$-dependent charges, but permanently charged sulfonate and quarternary ammonium groups. Complexes with these polyelectrolytes are made in the same way as described above. All rheological measurements are carried out on an Anton Paar MCR501 series stress-controlled rheometer using a cone-plate geometry.

The frequency sweeps shown in Fig. 1 are typical examples for our model polyelectrolyte complexes in a regime of linear deformation. The rheological response of polyelectrolyte complexes to an oscillatory strain depends strongly on salt concentration. At low salt concentration the response is similar to that of soft solids [8], whereas the response is liquidlike at high salt concentration. These observations are in line with results on biological electrostatic complexes [11]. 


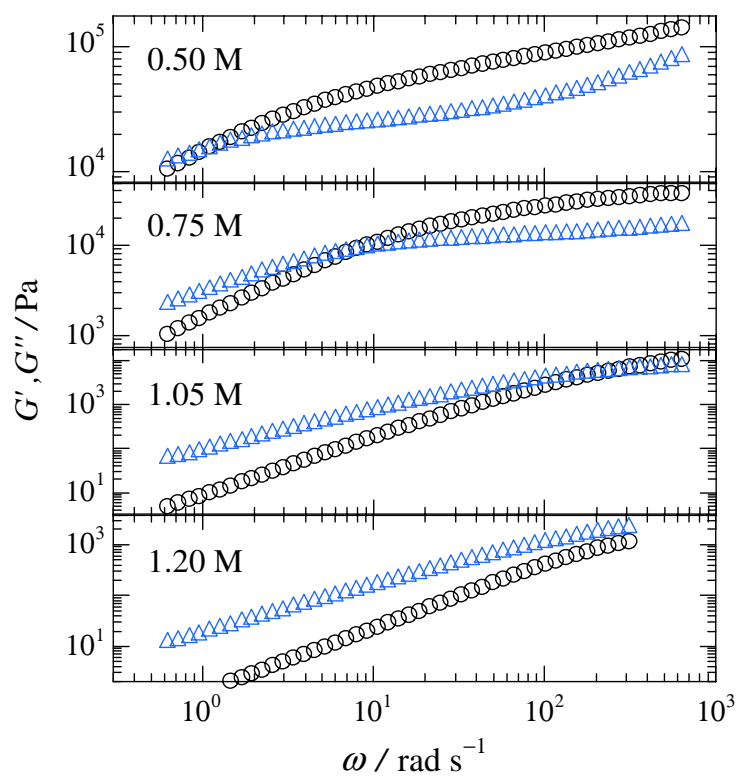

FIG. 1 (color online). Frequency sweeps of a $N=510$ polyelectrolyte complex at $\gamma=2 \%$ and salt concentrations as indicated in the figure, showing storage $(O)$ and loss $(\triangle)$ moduli.

Salt is known to accelerate dynamic processes in polyelectrolyte complexes. This leads to faster equilibration and eventually to hysteresis-free cycles of compression and disruption of polyelectrolyte complexes, as was shown using atomic force microscopy [12]. In well-known superposition methods in rheology, like time-temperature superposition and strain-rate-frequency superposition, such acceleration of dynamic processes provides the basis for probing dynamic processes on a range of time scales that is otherwise experimentally inaccessible. Could we use analogous rescaling of measurements shown in Fig. 1 in order to get more insight into the dynamics of these polyelectrolyte complexes as well? Figure 2(a) shows that the frequency sweeps for different salt concentrations can indeed be rescaled onto a single mastercurve using salt concentration dependent shift factors for the frequency, such that $\omega_{\text {scaled }}=\omega \tau_{c}$, and for the moduli, such that $G_{\text {scaled }}^{\prime}=$ $G^{\prime} / G_{c}$ and $G_{\text {scaled }}^{\prime \prime}=G^{\prime \prime} / G_{c}$. Shift factors $\tau_{c}$ and $G_{c}$ are chosen such that the crossover between storage and loss modulus, which is related to a typical relaxation time in many rheological models, occurs at a rescaled frequency and modulus of unity. Figure 2(a) shows that the relevant dynamic processes in these polyelectrolyte complexes respond similarly to salt concentration, which enables us to probe these complexes over seven decades in frequency.

The limits of the accessible dynamic range are dictated by a combination of instrumental and thermodynamic factors. The upper limit is given by the maximum accessible frequency of the rheometer and a theoretical minimum salt concentration close to zero. The lower limit is given by the critical salt concentration, beyond which polyelectrolyte complexes no longer form. In this case, we use a creep

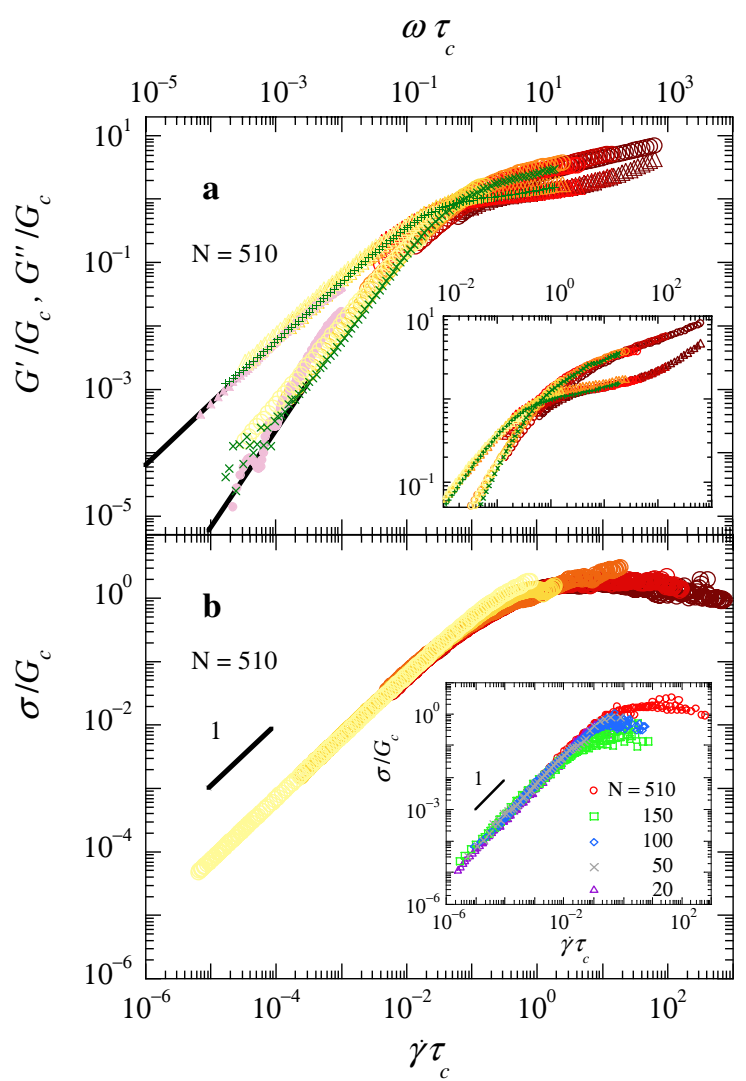

FIG. 2 (color online). (a) Rescaled frequency sweep of a $N=$ 510 polyelectrolyte complex at $\gamma=2 \%$. Storage moduli are shown using circles and crosses, loss moduli using triangles and pluses. Filled symbols are converted from a creep test. Solid lines are drawn to guide the eye. (b) Rescaled flow curves for $N=510$ polyelectrolyte complexes. The inset shows rescaled flow curves for $N=20(\triangle), 50(\times), 100(\diamond), 150(\square)$, and $510(\bigcirc)$ polyelectrolyte complexes. For reasons of clarity, one per five data points is shown in the inset.

test to probe the lowest frequencies and hence longest time scales. Data of creep compliance as a function of time are converted into frequency-dependent storage and loss moduli using a direct mathematical procedure [13].

If the apparent relaxation times, $\tau_{c}$, and moduli, $G_{c}$, are to provide a meaningful measure for the relevant dynamic processes in electrostatic complexes, we expect that they can be used to rescale nonlinear flow experiments as well. Indeed, Fig. 2(b) shows that both linear and nonlinear regimes of strain rate sweeps at various salt concentrations can be rescaled using the same shift factors as for Fig. 2(a). The flow curves all start with a Newtonian regime, followed by a nonlinear regime of strong shear thinning, which becomes more pronounced at low salt concentrations. This regime is characterized by large intermittent stress fluctuations, reminiscent of other associative networks [14], for which the steady state is taken as the average.

As shown in the inset of Fig. 2(b), a similar rescaling can also be applied to polyelectrolytes of different chain 
lengths, and even to a different set of polyelectrolytes $(N=100)$. A close inspection of the curves reveals that the onset of the nonlinear regime is not exactly the same for all chain lengths. This can be explained by the fact that polymer concentrations in these complexes are not completely independent of chain length [9], and entanglements become important for longer polymers.

Our observation that both linear and nonlinear rheological data can be superimposed by using two salt-dependent shift factors, $\tau_{c}$ and $G_{c}$, suggests that a simple mechanism underlies the dynamics of electrostatic complexes. Figure 3 shows that there is a strong dependence of the apparent relaxation time, $\tau_{c}$ and a much weaker dependence of the modulus, $G_{c}$, on salt concentration for all chain lengths.

The depicted dependences of $\tau_{c}$ and $G_{c}$ are closely related to the stress relaxation mechanism inside polyelectrolyte complexes. These complexes can be seen as concentrated two-component polymer solutions. Stress relaxation occurs by movement of chains, which is hindered by associations in the form of ion-ion pairs between two oppositely charged polyelectrolytes (sticky points). This suggests that the dynamics of chains with "sticky points" should apply, for which [15]

$$
\tau_{c} \approx N^{\alpha} \phi_{p}^{\beta} \zeta_{0}\left(c_{\text {salt }}\right)
$$

where $N$ is the polymer chain length, $\phi_{p}$ is the polymer volume fraction and $\zeta_{0}$ is an effective friction coefficient, which depends on salt concentration. The magnitude of $\alpha$ depends on the regime of relaxation: for Rouse-like relaxation $\alpha=2$, for reptation $\alpha=3.4$. The magnitude of $\beta$ depends somewhat on solvent quality, but is typically close

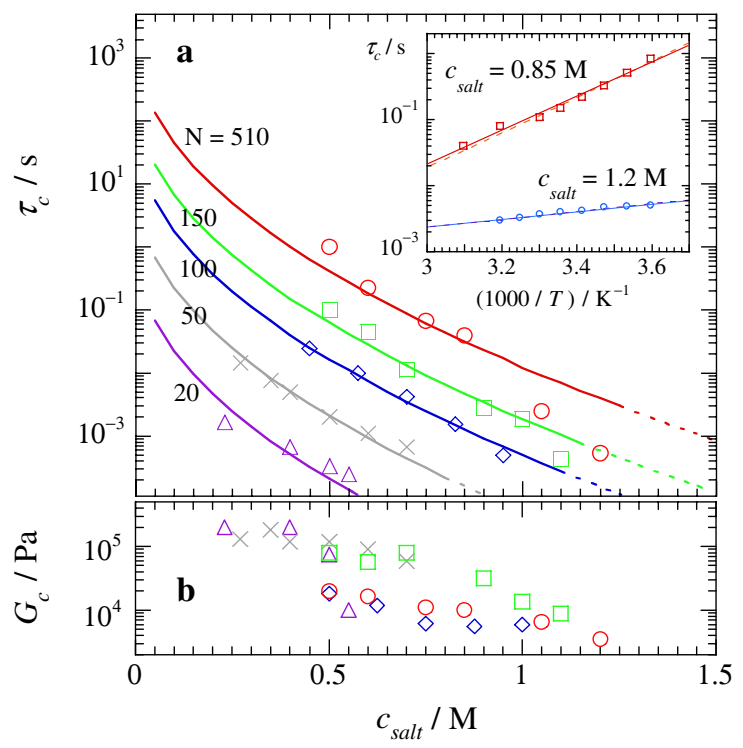

FIG. 3 (color online). (a) Relaxation times and (b) moduli shift factors, for rheological data of polyelectrolyte complexes with $N=20(\triangle), 50(\times), 100(\diamond), 150(\square)$, and $510(\bigcirc)$. The solid lines in (a) are fits to Eq. (3); the inset shows relaxation times for different temperatures at two indicated salt concentrations. to unity [15]. The breaking of association points is related to the dissociation rate of ion-ion pairs. We assume this is an activated process, governed by an energy barrier, $E_{a}$

$$
\zeta_{0}\left(c_{\text {salt }}\right)=\frac{1}{\omega_{0}} \exp \left[\frac{E_{a}\left(c_{\text {salt }}\right)}{k_{B} T}\right] .
$$

Reorganization of associations encompasses the breaking of polymeric ion-ion pairs, separating each two ionic groups, thereby surrounding them with monovalent counterions, and reforming polymeric ion-ion pairs at different positions. This route contains an energy barrier that can be estimated as the difference between the electrical free energy of four separated ionic groups of charge $\pm e$ in an electrolyte solution, and the Coulombic energy of two ion-ion pairs in contact: $E_{a} \approx E_{\text {corr }}-E_{\text {Coul }}$. The electrical correlation energy is approximated as $E_{\text {corr }} \approx$ $-2 \kappa e^{2} / 4 \pi \epsilon \epsilon_{0} \propto \sqrt{c_{\text {salt }}}$ [16], where $\kappa$ is the inverse Debye length. The Coulombic energy can be written as $E_{\text {Coul }}=-e^{2} / 2 \pi \epsilon \epsilon_{0} d$, where $d$ is the contact distance of charged groups in an ion-ion pair. Combining this with Eqs. (1) and (2), we find

$$
\tau_{c} \propto \frac{1}{\omega_{0}} N^{\alpha} \phi_{p}^{\beta} \exp \left[-a(T) \sqrt{c_{\text {salt }}}+b(T)\right]
$$

where $c_{\text {salt }}$ is the ionic strength in $\mathrm{mol} / \mathrm{L}$ for a $1: 1$ electrolyte, $a(T)=\sqrt{2000 N_{A}} e^{3} / 2 \pi\left(\epsilon \epsilon_{0} k_{B} T\right)^{3 / 2}$ and $b(T)=$ $e^{2} / 2 \pi \epsilon \epsilon_{0} k_{B} T d$.

Equation (3) predicts that the activation barrier depends on the square root of salt concentration, leading to a stretched exponential dependence of $\tau_{c}$ on $c_{\text {salt }}$. We have previously found that also $\phi_{p}$ depends weakly on $c_{\text {salt }}$ [9]. This dependence is, however, significantly weaker than the variations in $\tau_{c}$ shown in Fig. 3. The relaxation times presented in Fig. 3(a) have therefore been fitted to Eq. (3), using only an adjustable prefactor $\left\{A=\omega_{0}^{-1} N^{\alpha}\right.$ $\left.\phi_{p}^{\beta} \exp [b(T)]\right\}$, and a fixed value of 11 for $a(T)$. We find an excellent agreement between our data and the presented model. Moreover, the value of $a(T)$ we find is quantitatively in agreement with the theoretical prediction of 11.3 for $\epsilon=45$ and $T=293 \mathrm{~K}$, which is a reasonable assumption for concentrated polyelectrolyte complexes [17]. Finally, the prefactors, $A$, scale according to $N^{\alpha}$, with $\alpha=2.5$, as expected.

A key assumption in our conceptual picture is that an activated dissociation of ion-ion pairs governs the relaxation dynamics of electrostatic complexes. Consequently, $\tau_{c}$ should be a function of temperature as well. This is verified in a time-temperature superposition experiment, where we determine relaxation times as a function of temperature [Fig. 3(a), inset]. Assuming that $\epsilon$ is independent of $T$ [17], we expect $\tau_{c}$ to scale with $\exp \left(T^{-1}\right)$ or $\exp \left(T^{-1.5}\right)$ as the two limiting cases [Eq. (3))]. The fits in the inset of Fig. 3(a) for $\exp \left(T^{-1}\right)$ (solid lines) and $\exp \left(T^{-1.5}\right)$ (dashed lines), show that it is experimentally not possible to distinguish between these limiting cases for aqueous systems. 


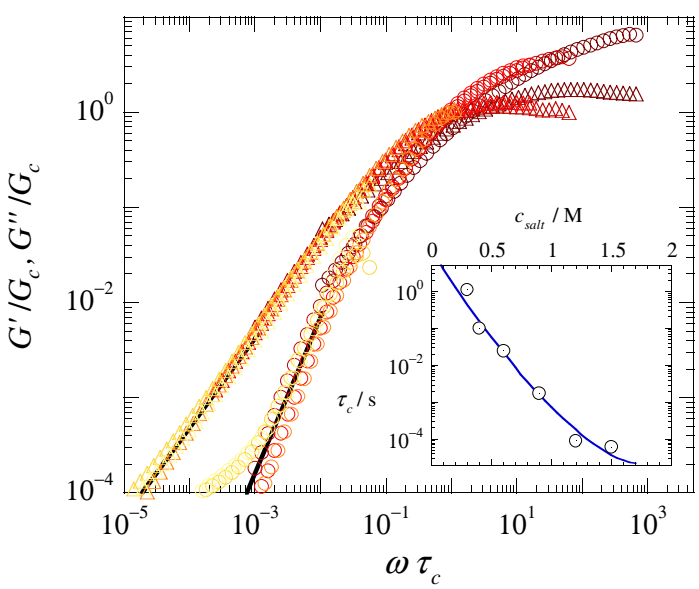

FIG. 4 (color online). Rescaled frequency sweeps of a telechelic network of PSPMA ${ }_{28} \mathrm{PEO}_{230} \mathrm{PSPMA}_{28}$ and $\mathrm{PAH}_{171}$ at $\gamma=5 \%$. Storage moduli are shown using circles; loss moduli using diamonds. The inset shows $\tau_{c}$ as a function of $c_{\text {salt }}$.

In contrast to the relaxation times, the moduli are found to depend only weakly on $c_{\text {salt }}$. This is simply due to the coupling between salt concentration and overall polymer density, as was shown in [9]. We find a linear relation between the modulus and $\phi_{p} / N$ (data not shown), as expected for unentangled polymer solutions in which each chain contributes $k_{B} T$ to the modulus [15].

The presented analysis of the dynamics in polyelectrolyte complexes provides a general tool to study dynamics in other soft materials that are assembled by electrostatic complexation. To verify this generality, we carry out the same approach on a transient network formed by telechelic polymers [5]. This network is made from ABA triblock copolymers with charged end blocks that are complexed with oppositely charged homopolymers. Electrostatic complexes form the nodes of this network, while the neutral and soluble $B$ blocks form the links between the nodes. We find that frequency sweeps of these networks can be rescaled onto a single mastercurve as well, as shown in Fig. 4. Using superposition we can access over eight decades in frequency and obtain the typical relaxation times at different salt concentrations.

The dynamics of electrostatically assembled materials is strongly dependent on salt concentration. A detailed understanding of these effects requires an effective method to probe the widely varying dynamic behavior of electrostatic assemblies at different salt concentrations. By rescaling rheological data, one can probe linear and nonlinear mechanical properties of these materials over a wide range of, otherwise inaccessible, time scales and study the salt dependence of relaxation mechanisms. This approach offers new ways to elucidate unresolved issues in the dynamics of electrostatic complexes: for example, those concerning the formation mechanism of electrostatic complexes [18] and those concerning the dynamics of biological complexes, such as the DNA-histone complex, at rest or under mechanical and chemical stresses during cell division [19].

The authors gratefully acknowledge Frans Leermakers for fruitful discussions. E.S. and J.S. acknowledge the Netherlands Organisation for Scientific Research (NWO) for financial support.

*evan.spruijt@wur.nl

[1] C. Herold, P. Schwille, and E. P. Petrov, Phys. Rev. Lett. 104, 148102 (2010).

[2] M. Sankaram and D. Marsh, Protein-Lipid Interactions (Elsevier, New York, 2001).

[3] M. Perutz, Science 201, 1187 (1978).

[4] M.E. Leunissen, C. G. Gristova, A.-P. Hynninen, C.P. Royall, A. I. Campbell, A. Imhof, M. Dijkstra, R. van Roij, and A. van Blaaderen, Nature (London) 437, 235 (2005).

[5] M. Lemmers, J. Sprakel, I. Voets, J. van der Gucht, and M. Cohen Stuart, Angew. Chem., Int. Ed. 49, 708 (2010).

[6] M. A. Cohen Stuart, B. Hofs, I. K. Voets, and A. de Keizer, Curr. Opin. Colloid Interface Sci. 10, 30 (2005).

[7] J. Ferry, Viscoelastic Properties of Polymers (John Wiley \& Sons, New York, 1980), 3rd ed..

[8] H. M. Wyss, K. Miyazaki, J. Mattsson, Z. Hu, D. R. Reichman, and D. A. Weitz, Phys. Rev. Lett. 98, 238303 (2007).

[9] E. Spruijt, A. H. Westphal, J.W. Borst, M. A. Cohen Stuart, and J. van der Gucht, Macromolecules 43, 6476 (2010).

[10] E. Spruijt, J. Sprakel, M. A. C. Stuart, and J.v. d. Gucht, Soft Matter 6, 172 (2010).

[11] R. C. Liu, Y. Morishima, and F. Winnik, Polym. J. 34, 340 (2002).

[12] E. Spruijt, M.A. Cohen Stuart, and J.v.d. Gucht, Macromolecules 43, 1543 (2010).

[13] R. M. L. Evans, M. Tassieri, D. Auhl, and T. A. Waigh, Phys. Rev. E 80, 012501 (2009).

[14] J. Sprakel, E. Spruijt, J. van der Gucht, J. Padding, and W. Briels, Soft Matter 5, 4748 (2009).

[15] M. Rubinstein and A. N. Semenov, Macromolecules 34, 1058 (2001).

[16] T. Hill, An Introduction to Statistical Thermodynamics (Dover, New York, 1986).

[17] D. Loginova, A. Lileev, and A. Lyashchenko, Russ. J. Inorg. Chem. 47, 1426 (2002).

[18] G. C. Wong, J. X. Tang, A. Lin, Y. Li, P. Janmey, and C. R. Safinya, Science 288, 2035 (2000).

[19] J. F. Marko and M. G. Poirier, Biochem. Cell Biol. 81, 209 (2003). 